\title{
Hereditary nonpolyposis colorectal cancer: pitfalls in deletion screening in MSH2 and MLH1 genes
}

\author{
Maria Wehner ${ }^{1}$, Elisabeth Mangold ${ }^{1}$, Marlies Sengteller ${ }^{1}$, Nicolaus Friedrichs ${ }^{2}$, Stefan Aretz ${ }^{1}$, \\ Waltraut Friedl $^{1}$, Peter Propping ${ }^{1}$ and Constanze Pagenstecher*,1
}

\author{
${ }^{1}$ Institute of Human Genetics, University of Bonn, Bonn, Germany; ${ }^{2}$ Institute of Pathology, University of Bonn, Bonn, \\ Germany
}

Hereditary nonpolyposis colorectal cancer (HNPCC) is caused by a deficiency in DNA mismatch repair in consequence of germline mutations mainly in the genes $M S H 2$ and $M L H 1$. Around $10 \%$ of patients suspected of HNPCC are identified with large genomic deletions that cannot be detected by conventional methods of mutation screening. The recently developed multiplex ligation-dependent probe amplification (MLPA) proved to be an easy to perform method for deletion detection and is reliable when more than one exon is deleted. We show that, in some cases, apparent deletions of single exons may actually result from single base substitutions or small insertions/deletions in the hybridisation sequence of MLPA probes. We conclude that single exon deletions, detected by MLPA or multiplex PCR, should be validated with additional methods. European Journal of Human Genetics (2005) 13, 983-986. doi:10.1038/sj.ejhg.5201421;

published online 4 May 2005

Keywords: hereditary nonpolyposis colorectal cancer; HNPCC; MSH2; MLH1; deletion screening; MLPA

\section{Introduction}

Hereditary nonpolyposis colorectal cancer (HNPCC) is an autosomal-dominant disease characterised by a predisposition to early onset colorectal cancer and other tumours. ${ }^{1,2}$ It is caused by germline mutations in DNA mismatch repair (MMR) genes, mainly MSH2 and MLH1. Most of the published mutations are single base substitutions, small deletions or insertions leading to altered MMR proteins (InSIGHT mutation data base; http://www.insight-group. org). A high proportion of large genomic deletions in the MSH2 gene detected by use of Southern blot technique was first reported in Dutch HNPCC patients. ${ }^{3}$ The development of novel, PCR-based techniques for deletion screening including semiquantitative multiplex PCR and multiplex ligation-dependent probe amplification (MLPA) has considerably simplified screening for large genomic deletions

*Correspondence: Dr C Pagenstecher, Institute of Human Genetics, University of Bonn, Wilhelmstrasse 31, 53111 Bonn, Germany.

Tel: + 49228287 2342; Fax: + 49228287 2380;

E-mail: Constanze.Pagenstecher@ukb.uni-bonn.de

Received 28 December 2004; revised 23 February 2005; accepted 3 March 2005; published online 4 May 2005 in DNA mismatch repair genes. ${ }^{4-6}$ Consequently, various patient cohorts have been screened for large genomic deletions in MSH2 and MLH1 genes. Depending on the patient selection criteria, deletions have been reported in up to $13 \%$ of patients suspected of HNPCC. ${ }^{7-10}$ Considering the high frequency of deletions and the fact that MLPA is rapid and easy to perform, ${ }^{9}$ deletion screening was proposed as a first step of mutation analysis in HNPCCsuspects prior to the more labour-intensive exon-by-exon screening for point mutations. However, as demonstrated by the following examples, in some cases, the results of deletion screening have to be interpreted with caution and should be verified with additional methods.

\section{Materials and methods}

Patients

The patients included in the study were recruited at the University Hospital, Bonn in accordance with the study protocol of the German HNPCC Consortium. ${ }^{11}$ Informed written consent was obtained from all patients included in the study. The study was approved by the ethical committee of the University Hospital Bonn. 
Detection of large deletions on genomic DNA

Genomic DNA was extracted from peripheral EDTA-anticoagulated blood samples according to the standard saltingout procedure. Exon deletions in the MSH2 or MLH1 genes of HNPCC patients were identified by semiquantitative multiplex PCR or MLPA. Multiplex PCR of all 35 exons of MSH2 and MLH1 genes was performed in seven PCR reactions, each containing six pairs of primers as described. ${ }^{5}$ In MLPA, each probe consists of two oligonucleotides that hybridise to adjacent sites of the target sequence; hybridised probe oligonucleotides are ligated, permitting subsequent amplification. All ligated probes have identical end sequences, permitting simultaneous PCR amplification by use of only one pair of primers. ${ }^{4}$ With the MLPA test (SALSA P003 MLH1/MSH2, MRC Holland), probes for all 16 exons of MSH2, 19 exons of MLH1, and seven control probes from other chromosomal regions can be amplified in a single PCR reaction. The FAM-labelled PCR products were separated on an ABI 377 or an ABI 3100 (Applied Biosystems) sequencer and analysed by Genotyper or Genemapper software (Applied Biosystems). Semiquantitative evaluation of electropherograms was performed by visual comparison of the peak heights of each $\mathrm{MSH} 2$ or MLH1 exon in the patient sample and the corresponding exon in a normal control. Each deletion detected was confirmed in a second independent MLPA reaction.

\section{Transcript analysis}

Fresh venous blood samples $(2.5 \mathrm{ml})$ were collected into PAXgene Blood RNA Tubes (PreAnalytiX, Qiagen, Hilden, Germany) containing RNA stabilising solution. Total RNA was extracted by use of the PAXgene Blood RNA Kit (Qiagen, Hilden, Germany) according to the manufacturer's protocol. First strand cDNA was synthesised from 2 to $3 \mu \mathrm{g}$ of total RNA by random hexamer-primed reverse transcription with the Superscript 1st strand system for RT-PCR (Invitrogen GmbH, Karlsruhe, Germany) according to the manufacturer's protocol. RT-PCR fragments were obtained according to standard PCR protocols by use of different primers to generate the appropriate fragments (primer sequences see Table 1). RTPCR products were separated on a 1\% agarose gel and visualised with ethidium bromide. Gels containing different
RT-PCR fragments were examined on an UV imaging system. Individual bands were excised from the gel and eluted by use of the High Pure PCR Product Purification Kit (Roche Diagnostics, Mannheim, Germany). Eluted DNA was reamplified with the same pairs of primers and sequenced on an ABI Prism 377 or ABI 3100 automated sequencer (Applied Biosystems) using cycle sequencing procedure and the BigDye terminator kit version 2.0 or 1.1, respectively.

\section{Confirmation of genomic deletions}

Long-range PCR on genomic DNA was used to confirm the deletions uncovered by multiplex PCR and/or MLPA. With the Expand High Fidelity PCR System (Roche Diagnostics, Mannheim, Germany), primers located in the introns $5^{\prime}$ and $3^{\prime}$ to the nondeleted exons flanking the deleted exons were applied. PCR was performed according to the manufacturer's recommendations with some modifications as described by Wang et al. ${ }^{5}$ PCR products were separated on a $1 \%$ agarose gel and visualised by ethidium bromide staining.

To determine the breakpoints of deletions, long-range PCR products containing the expected deletions were cut out from the gel, purified by QIAquick PCR purification kit (Qiagen, Hilden, Germany) and sequenced by cycle sequencing.

\section{Results}

Screening for large genomic deletions by use of MLPA pointed to a deletion of MLH1 exon 13 in patients 826 and 981 (Figure 1a). Confirmation of the deletion in genomic DNA by long-range PCR is difficult, since the introns 12 and 13 of the MLH1 gene are quite large (2776 and $11253 \mathrm{bp}$, respectively). Therefore, we examined mRNA of the patients to confirm the deletion. cDNA was amplified with a forward primer in exon 12 and a reverse primer in exon 15 and PCR products were separated on an agarose gel. An additional fragment of $385 \mathrm{bp}$ pointing to a deletion of exon 13 was detected only in patient 826 (Figure 1b). Sequencing of the PCR product confirmed the deletion of exon 13 in MLH1 in patient 826 (Figure 1c). The deletion of exon 13 detected by MLPA in patient 981 was not confirmed by cDNA analysis (Figure $1 \mathrm{~b}$ and $\mathrm{c}$ ). However, sequencing of genomic DNA or cDNA of patient

Table 1 Primer sequences used for confirmation of deletions on mRNA or DNA level

\begin{tabular}{|c|c|c|c|}
\hline Gene & Exon/intron & Primer sequences & $\begin{array}{l}\text { Reference sequences } \\
\text { and location of primers (nt positions) }\end{array}$ \\
\hline MLH1 & $\begin{array}{l}\text { Exon } 12 \mathrm{~F} \\
\text { Exon } 15 \mathrm{R}\end{array}$ & $\begin{array}{l}\text { 5'-GATGGTTCGTACAGATTCCCG-3' } \\
\text { 5'-GAGTATCTGGTAGAACAGTTC-3' }\end{array}$ & $\begin{array}{l}\text { NM_000249: } 1206-1226 \\
\text { NM_000249: } 1752-1732\end{array}$ \\
\hline MSH2 & $\begin{array}{l}\text { Exon } 12 \mathrm{~F} \\
\text { Exon } 15 \mathrm{R}\end{array}$ & $\begin{array}{l}\text { 5'-CTTGTTAACCAGTGGATTAAGCAG-3' } \\
\text { 5'-GTTTTACAAGGAATTCATGGTITT-3' }\end{array}$ & $\begin{array}{l}\text { NM_000251: } 1089-1112 \\
\text { NM_000251: } 1482-1458\end{array}$ \\
\hline MSH2 & $\begin{array}{l}\text { Intron 7F } \\
\text { Intron 8R }\end{array}$ & 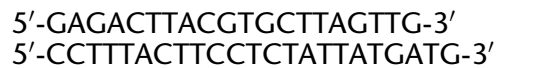 & $\begin{array}{l}\text { AC009600: } 32820-32839 \\
\text { AC009600: } 55848-55826\end{array}$ \\
\hline
\end{tabular}




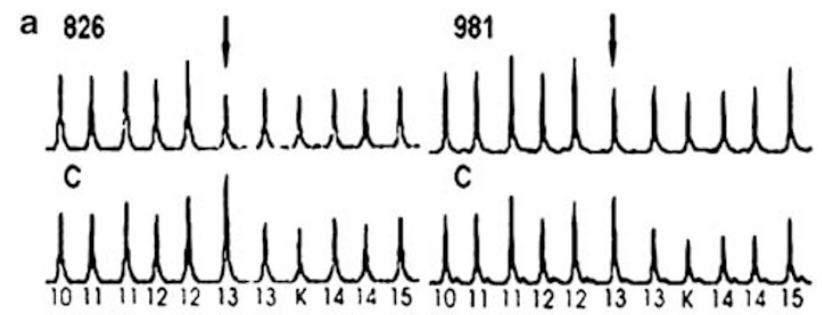

b

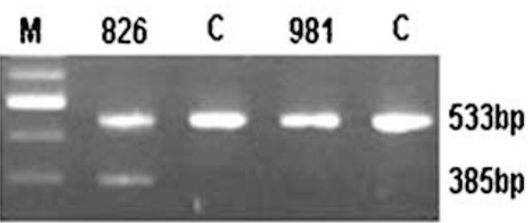

C

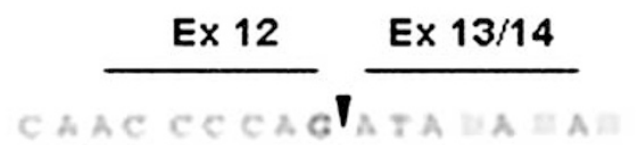

826
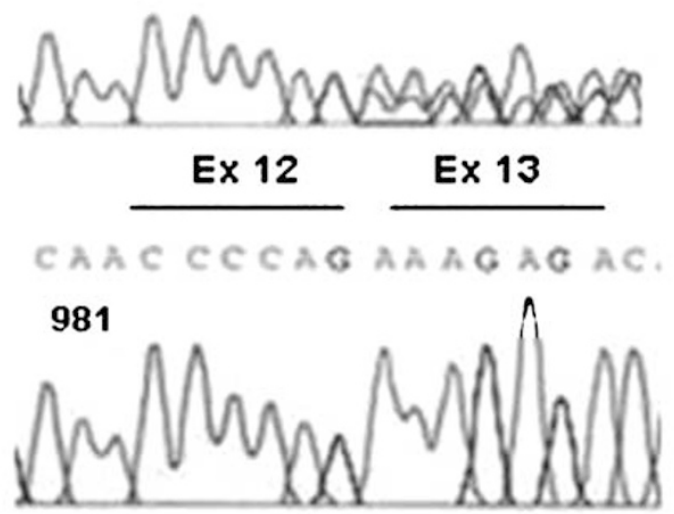

Figure 1 Characterisation of deletions of MLH1 exon 13. (a) MLPA analysis pointing to a deletion of exon 13 of the $M L H 1$ gene in patients 826 and 981 (arrows); C: controls. (b) Agarose gel showing RT-PCR products obtained on CDNA of patients 826 and 981 and two controls by use of a forward primer in exon 12 and a reverse primer in exon 15 . An additional $385 \mathrm{bp}$ fragment due to a deletion of exon 13 was observed only in patient 826 . M-DNA ladder with a spacing of $100 \mathrm{bp}$. (c) Sequencing pattern of the exons $12-13 / 14$ junction of RT-PCR products of patients 826 and 981 . A deletion of exon 13 is confirmed only in patient 826. Exon 13 is not deleted in patient 981 .

981 revealed a single base substitution (c.1459C $>$ T; p.Arg487X) localised within the hybridisation sequence of the MLPA probe for exon 13, $9 \mathrm{bp}$ upstream of the ligation site (data not shown).

A similar situation was found in patient 1041. In this case, the MLPA test indicated a deletion of MLH1 exon 2. Sequencing of exon 2 revealed a nonsense mutation (c.184C > T; p.Gln62X) localised $1 \mathrm{bp}$ before the ligation site of the MLPA probes for exon 2 (data not shown).

In patient 518 , a deletion of exon 8 of the MSH2 gene was detected by use of multiplex PCR as described (Figure 2a).
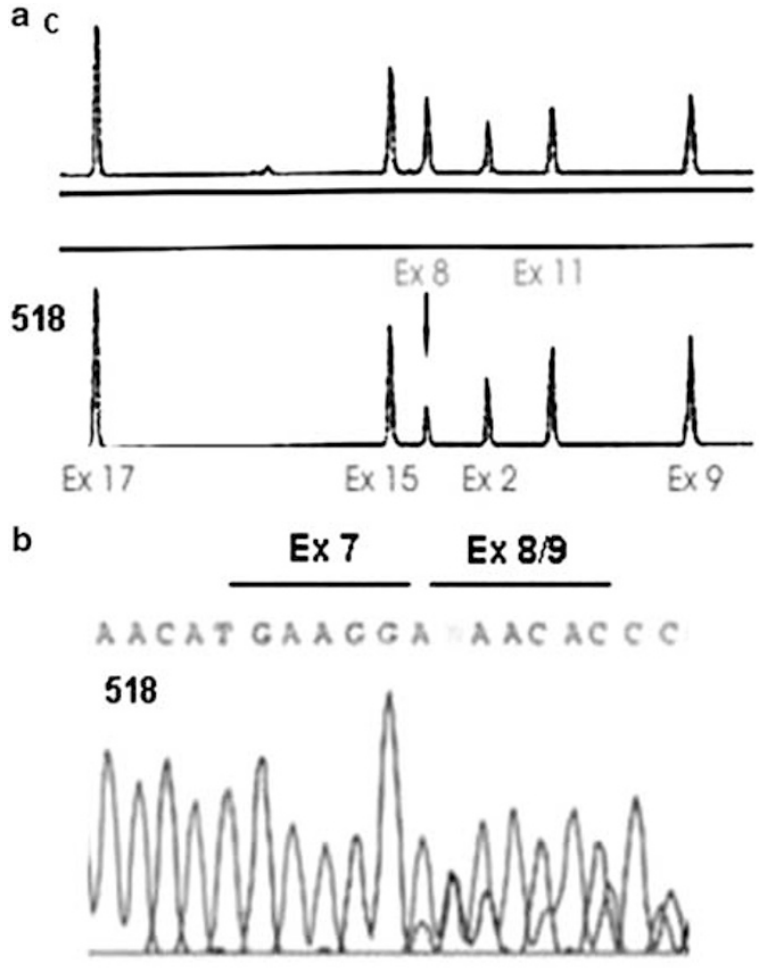

C

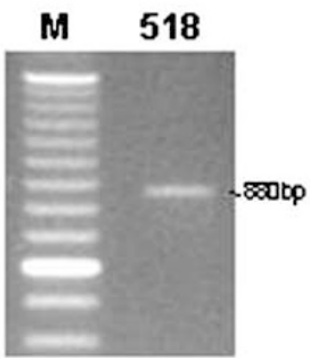

Figure 2 Characterisation of the deletion of $M S H 2$ exon 8 in patient 518. (a) Deletion of exon 8 in the MSH2 gene detected by use of the multiplex PCR method. C: control. (b) Sequencing pattern of the exons 7-8/9 junction of RT-PCR products showing a deletion of exon 8. (c) Agarose gels showing the long range PCR product obtained with primers in intron 7F and intron 8R.

First experiments failed to confirm this deletion in genomic DNA by long-range PCR. ${ }^{8}$ Later, the deletion was also detected by MLPA and confirmed by cDNA analysis (Figure $2 \mathrm{~b}$ ). The breakpoints of this deletion could be determined by further examinations of genomic DNA with primers localised in introns 7 and 8 of the MSH2 gene (Table 1). This deletion comprises $22170 \mathrm{bp}$ of the MSH2 gene (nt.33 446-nt.55 616; GenBank AC009600) with both breakpoints localised within repetitive Alu sequences in intron 7 and 8, respectively.

While single exon deletions localised inside the genes, that is, with known borders, can usually be confirmed by RT-PCR analysis, it is more difficult to apply this approach when a deletion is localised in the first or last exon of the 
gene. By use of MLPA we detected a deletion of MSH2, exon 1 in two patients. Sequencing of this exon in genomic DNA revealed no changes in both patients; this indirectly supports the conclusion that the deletions are real. No long-range PCR product was obtained with different forward primers localised in the $5^{\prime}$ untranslated region of the gene. In one of the families (No. 368), the deletion of MSH2 exon 1 was detected in the index patient and in her son. Both patients also carried a rare missense mutation in MSH2, exon 5 (c.847G > T; p.Asp283Tyr). It was shown by mRNA analysis that only one allele containing the normal sequence in exon 5 was present in the transcript. This approach indicates that both variations are localised on the same allele. The result indirectly confirms the deletion of exon 1 by demonstrating that the allele with the deletion of exon 1 is not transcribed.

\section{Discussion}

Results of the MLPA test (or other methods applied for deletion analysis) are usually correctly interpreted when two ore more adjacent exons are deleted. However, our data show that when only one exon appears to be deleted the result has to be interpreted with caution and should be verified by an additional method.

By use of MLPA we identified a deletion of one exon in the MLH1 gene in three patients. Only one of these deletions could be confirmed on the cDNA level. In fact, the other two were due to point mutations localised in the hybridisation sites of the corresponding MLPA probes.

To avoid misinterpretations of the results by MLPA screening, a solution would be to include two different sets of probes for each exon. Sequences with known polymorphisms should be excluded as hybridisation sites. The correct interpretation of the results of deletion analysis is of great importance in hereditary tumour diseases such as HNPCC where a mutation detected in the index patient is used for predictive diagnostics. An apparent deletion of a single exon detected by MLPA screening might in truth be: (a) A true deletion, such as in patient 518. In this case predictive testing would be correct with any method able to detect the deletion. (b) No deletion at all, but a harmless polymorphism situated in the hybridisation site. If one would falsely interpret this 'deletion' as the causative mutation in the index patient, any predictive testing based on this in the family using whatever method for deletion detection would lead to inconclusive results. (c) No deletion at all but a different pathogenic mutation, for example, in patients 981 and 1041. In this case, problems might arise when diagnostic procedures applied on the index patient differ from those applied on persons at risk. Predictive diagnostics based on a 'deletion' might lead to a false test result when methods different from MLPA are applied for deletion testing. In this respect, it is advisable to apply the same test on both the index patient and the person at risk in a given family. When predictive testing is performed by another laboratory and in the absence of the index patient's DNA, it is necessary to report in detail the method by which a mutation or deletion had been detected.

\author{
Databases: \\ HNPCC - OMIM 114500 \\ MSH2 - OMIM: 120435; GDB: 203983; GenBank: \\ NM_000251; AC009600 \\ MLH1 - OMIM: 120436; GDB: 249617; GenBank: \\ NM_000249 \\ http://www.nfdht.nl (ICG-HNPCC mutation database)
}

\section{Acknowledgements}

We thank the patients who have participated in this study. The work of the German HNPCC Consortium is supported by a multicentre grant from the Deutsche Krebshilfe, Bonn, Germany, Project No.: 70-3027Ma1.

\section{References}

1 Vasen HF, Watson P, Mecklin JP, Lynch HT: New clinical criteria for hereditary nonpolyposis colorectal cancer (HNPCC, Lynch syndrome) proposed by the International Collaborative group on HNPCC. Gastroenterology 1999; 116: 1453-1456.

2 Lynch HT, de la Chapelle A: Hereditary colorectal cancer. N Engl J Med 2003; 348: 919-932.

3 Wijnen J, van der Klift $\mathrm{H}$, Vasen $\mathrm{H}$ et al: MSH2 genomic deletions are a frequent cause of HNPCC. Nat Genet 1998; 20: 326-328.

4 Schouten JP, McElgunn CJ, Waaijer R, Zwijnenburg D, Diepvens F, Pals G: Relative quantification of 40 nucleic acid sequences by multiplex ligation-dependent probe amplification. Nucleic Acids Res 2002; 30: e57.

5 Wang Y, Friedl W, Sengteller M et al: A modified multiplex PCR assay for detection of large deletions in MSH2 and MLH1. Hum Mutat 2002; 19: 279-286.

6 Charbonnier F, Raux G, Wang Q et al: Detection of exon deletions and duplications of the mismatch repair genes in hereditary nonpolyposis colorectal cancer families using multiplex polymerase chain reaction of short fluorescent fragments. Cancer Res 2000; 60: 2760-2763.

7 Charbonnier F, Olschwang S, Wang Q et al: MSH2 in contrast to MLH1 and MSH6 is frequently inactivated by exonic and promoter rearrangements in hereditary nonpolyposis colorectal cancer. Cancer Res 2002; 62: 848-853.

8 Wang Y, Friedl W, Lamberti C et al: Hereditary nonpolyposis colorectal cancer: frequent occurrence of large genomic deletions in MSH2 and MLH1 genes. Int J Cancer 2003; 103: 636-641.

9 Gille JJ, Hogervorst FB, Pals G et al: Genomic deletions of MSH2 and MLH1 in colorectal cancer families detected by a novel mutation detection approach. Br J Cancer 2002; 87: 892-897.

10 Taylor CF, Charlton RS, Burn J, Sheridan E, Taylor GR: Genomic deletions in MSH2 or MLH1 are a frequent cause of hereditary non-polyposis colorectal cancer: identification of novel and recurrent deletions by MLPA. Hum Mutat 2003; 22: 428-433.

11 Mangold E, Pagenstecher C, Friedl W et al: Spectrum and frequencies of mutations in MSH2 and MLH1 identified in 1721 German families suspected of hereditary nonpolyposis colorectal cancer. Int J Cancer 2005, (in press). 